\title{
Assessment of the hydrothermal resistance of Y-TZP ceramics by the degree of tetragonality of major phases
}

\author{
O.S. Tolkachev ${ }^{\dagger}$, E. S. Dvilis, T. R. Alishin, O. L. Khasanov, D. A. Miheev, T. Chzhan \\ ${ }^{\dagger}$ ost4@tpu.ru
}

Tomsk Polytechnic University, 30 Lenin Av., Tomsk, 634034, Russia

\begin{abstract}
Ceramic and composite materials based on Y-TZP and metastable $\mathrm{Al}_{2} \mathrm{O}_{3}$ nanofibers were manufactured by uniaxial single-axis pressing followed by free sintering in the temperature range of $1350-1600^{\circ} \mathrm{C}$. The addition of $1 \mathrm{vol} . \% \mathrm{Al}_{2} \mathrm{O}_{3}$ nanofibers makes it possible to produce ceramics with $196 \mathrm{~nm}$ grain and a relative density of $98 \%$ at $1400^{\circ} \mathrm{C}$. For samples without nanofibers, the density value approaches $98 \%$ only at a sintering temperature of $1500^{\circ} \mathrm{C}$ and is accompanied by an increase in the average grain size by $25 \%$. It was found that the introduction of $\mathrm{Al}_{2} \mathrm{O}_{3}$ nanofibers into $\mathrm{Y}$-TZP is accompanied by an increase in hydrothermal resistance: the content of the monocline phase after 40 hours of testing at $134^{\circ} \mathrm{C}$ water vapour temperature and a pressure of $0.2 \mathrm{MPa}$ does not exceed $3.5 \mathrm{vol} . \%$ in samples manufactured in the sintering temperature range of $1400-1500^{\circ} \mathrm{C}$, while in samples without nanofibers sintered at $1500^{\circ} \mathrm{C}$, the content of the monocline phase after such tests exceeds $60 \%$. A regular relationship has been found between the degree of tetragonality the source material and the formation of the monoclinic phase after accelerated ageing. After the ageing test at a vapour temperature of $180^{\circ} \mathrm{C}$ and a pressure of $1 \mathrm{MPa}$, the phase content of the studied ceramics remains stable with the degree of tetragonality not exceeding 1.0145. The discovered regularity expands the possibilities for optimization of manufacturing modes and achievement of the required balance of various operating properties of Y-TZP ceramics used as implants or responsible products working in a humid environment.
\end{abstract}

Keywords: zirconia, alumina, hydrothermal resistance, degree of tetragonality.

УДК: 666.3

\section{Оценка гидротермальной стойкости керамики Y-TZP по степени тетрагональности основных фаз}

\author{
Толкачёв О. С. , Двилис Э. С., Алишин Т. Р., Хасанов О. Л., Михеев Д. А., Чжан Ц. \\ Томский политехнический университет, пр. Ленина, 30, Томск, 634034, Россия
}

\begin{abstract}
Методом одноосного одностороннего прессования с последующим свободным спеканием в диапазоне температур $1350-1600^{\circ} \mathrm{C}$ изготовлены керамические и композиционные материалы на основе Y-TZP и метастабильных нановолокон $\mathrm{Al}_{2} \mathrm{O}_{3}$. Добавление 1 об.\% нановолокон $\mathrm{Al}_{2} \mathrm{O}_{3}$ позволяет при температуре $1400^{\circ} \mathrm{C}$ получить керамику с зерном 196 нм и с относительной плотностью 98\%. У образцов без нановолокон значение плотности приближается к 98\% лишь при температуре спекания $1500^{\circ} \mathrm{C}$ и сопровождается увеличением среднего размера зерна на $25 \%$. Установлено, что введение в Y-TZP нановолокон $\mathrm{Al}_{2} \mathrm{O}_{3}$ сопровождается повышением гидротермальной стойкости: содержание моноклинной фазы после 40 ч испытаний при температуре водяного пара $134^{\circ} \mathrm{C}$ и давлении $0.2 \mathrm{MПа}$ не превышает 3.5 об.\% в образцах, изготовленных в диапазоне температур спекания $1400-1500^{\circ} \mathrm{C}$, тогда как у образца без нановолокон, спеченного при $1500^{\circ} \mathrm{C}$, содержание моноклинной фазы после таких испытаний превышает $60 \%$. Обнаружена закономерная связь степени тетрагональности исходного материала с образованием в нём после ускоренного старения моноклинной фазы. После испытания на старение при температуре пара $180^{\circ} \mathrm{C}$ и давлении 1 МПа фазовый состав исследуемой керамики остаётся стабильным при степени тетрагональности не более 1.0145. Обнаруженная закономерность расширяет возможности для оптимизации режимов изготовления и достижения требуемого баланса различных эксплуатационных свойств керамики Y-TZP, используемой в качестве имплантатов или ответственных изделий, работающих во влажной среде.
\end{abstract}

Ключевые слова: диоксид циркония, оксид алюминия, гидротермальная стойкость, степень тетрагональности. 


\section{1. Введение}

Благодаря уникальным физическим и механическим свойствам диоксид циркония применяется в качестве материала для изготовления широкого класса изделий различного назначения: режущего инструмента, термобарьерных покрытий, твердооксидных топливных элементов, биоинертных имплантатов, в стоматологии для изготовления зубных коронок [1-5].

Стабилизированный иттрием тетрагональный поликристаллический диоксид циркония, известный как Y-TZP, обладает высокими механическими характеристиками: вязкость разрушения достигает $15 \mathrm{MПа} \cdot \mathrm{M}^{1 / 2}$, а предел прочности при изгибе - 1200 МПа [6,7]. Другими отличительными свойствами Y-TZP являются низкий коэффициент трения и высокая износостойкость, химическая инертность, а также низкая теплопроводность и высокая температура эксплуатации $[1,4,5,8]$.

Одним из немногих недостатков Y-TZР является склонность к деградации прочностных свойств под действием внешних факторов, обусловленная тетрагональномоноклинным фазовым переходом. Наиболее интенсивно деградация протекает во влажной среде при повышенных температурах и давлении, что существенно ограничивает области применения данного материала. Это явление известно как гидротермальное старение или LTD (lowtemperature degradation - низкотемпературная деградация) $[1,7,9-11]$.

Среди основных факторов, определяющих превращение тетрагональной в моноклинную фазу при старении, выделяют размер зерен, а также сегрегацию примесных и введённых в состав керамики ионов на границах структурных элементов [12-14]. Отмечается, что температура спекания более $1350^{\circ} \mathrm{C}$ приводит к неоднородному распределению ионов $\mathrm{Y}^{3+}$. Обеднённые ионами $\mathrm{Y}^{3+}$ кристаллиты выступают центрами зарождения моноклинной фазы [14].

Использование коммерческого наноразмерного порошка Y-TZP с 0.3 мол.\% $\mathrm{Al}_{2} \mathrm{O}_{3}$ (TZ-PX172, Tosoh Co., Япония) с высокой удельной поверхностью (17 м²/г), а также холодного изостатического прессования давлением 200 МПа позволило авторам работы [12] достигнуть значения плотности 99\% последующим спеканием при $1250^{\circ} \mathrm{C}$ в течение 2 ч. При этом, керамика практически не проявила признаков LTD после испытаний в воде при температуре $140^{\circ} \mathrm{C}$ и давлении 0.4 МПа: после испытаний в течении 1500 дней содержание моноклинной фазы не превысило 3\%. Однако для определения основного критерия старения - содержания моноклинной фазы - авторы данной работы использовали метод прямого сопоставления интенсивности рефлексов основных фаз керамики в узком диапазоне углов (от 26 до 33 градусов по 20), который не отличается высокой достоверностью и не позволяет раскрыть механизмы влияния среды на кристаллическую структуру Y-TZP.

Целью работы является изучение гидротермальной стойкости керамики Y-TZP с добавками нановолокон $\mathrm{Al}_{2} \mathrm{O}_{3}$, изготовленной статическим прессованием с последующим спеканием, а также выявление связи пара- метров кристаллической структуры составляющих фаз спечённой керамики с образованием в ней моноклинной фазы после гидротермального старения.

\section{2. Материал и методика исследований}

Исходным материалом керамической матрицы служил наноразмерный порошок $\mathrm{ZrO}_{2}+3$ мол.\% $\mathrm{Y}_{2} \mathrm{O}_{3}$ с удельной поверхностью $S_{\text {уд }}=7 \mathrm{~m}^{2} /$ г, (порошок марки TZ-3YS, Tosoh Co., Япония). Наполнителем являлись нановолокна $\mathrm{Al}_{2} \mathrm{O}_{3}\left(\mathrm{~S}_{\text {уд }}=90 \mathrm{~m}^{2} /\right.$ г), полученные методом жидкофазного каталитического окисления алюминия молекулярным кислородом при температуре $820^{\circ} \mathrm{C}$ (Fibrall, OCSiAl, Россия). Из исходных компонентов ультразвуковым диспергированием в этиловом спирте подготовили смесь с 1 об.\% нановолокон $\mathrm{Al}_{2} \mathrm{O}_{3}$. Выбор содержания $\mathrm{Al}_{2} \mathrm{O}_{3}$ обусловлен результатами предыдущих исследований, в ходе которых установлено, что использование метастабильных нановолокон $\mathrm{Al}_{2} \mathrm{O}_{3}$ способствует снижению температуры спекания, а в наибольшей степени этот эффект наблюдается на составе с 1 об.\% [15]. Значения теоретической плотности для Y-TZP и $\mathrm{Al}_{2} \mathrm{O}_{3}$ принимали 6.1 и 3.99 г/ см³ $^{3}$, соответственно.

Компактирование порошковых материалов осуществляли по схеме одноосного одностороннего прессования в стальной цилиндрической пресс-форме диаметром 10 мм при давлении 100 МПа. Масса навески составляла 0.5 г.

Свободное спекание спрессованных образцов высотой около 2.3 мм проводили в высокотемпературной печи LHT 02/18 (Nabertherm, Германия) при температурах в диапазоне от $1350^{\circ} \mathrm{C}$ до $1600^{\circ} \mathrm{C}$ с шагом $50^{\circ} \mathrm{C}$, с постоянной скоростью нагревания $200^{\circ} \mathrm{C} / ч$ и изотермической выдержкой в течение 1 ч. Плотность спеченных образцов оценивали методом гидростатического взвешивания в дистиллированной воде.

Аттестацию керамики до LTD-испытаний проводили методами рентгенофазового анализа (РФА) на дифрактометре XRD-7000S (Shimadzu, Япония) на отполированной поверхности в диапазоне углов (от $15^{\circ}$ до $70^{\circ}$ по $2 \theta$ ) с использованием метода Ритвельда в свободно распространяемом программном обеспечении "PowderCell". При уточнении использовали соответствующие PDF файлы тетрагональной фазы (PDF Card - 01-075-9648) и моноклинной (PDF Card - 00-065-0687).

Испытание керамики на стойкость к LTD проводили с использованием автоклава в парах воды при условиях, соответствующих требованиям двух стандартов: температура $134^{\circ} \mathrm{C}$ и давление $0.2 \mathrm{MПа} \mathrm{[16];} \mathrm{температура}$ $180^{\circ} \mathrm{C}$ и давление $1 \mathrm{MПа} \mathrm{[17].} \mathrm{Продолжительность}$ испытаний по первому стандарту (при $134^{\circ} \mathrm{C}$ ) была ограничена 40 часами; через каждые 10 часов испытаний проводили исследование результатов воздействия. Продолжительность испытаний по второму стандарту (при $180^{\circ} \mathrm{C}$ ) была ограничена 20 часами; через каждые 5 часов испытаний проводили исследование результатов воздействия. Согласно сведениям [1], 1 час ускоренного гидротермального старения при $134^{\circ} \mathrm{C}$ и давлении $0.2 \mathrm{MПа}$ условно соответствует 3-4 годам in vivo (при использовании данного материала в качестве имплантата). 
Первичную оценку относительного содержания моноклинной фазы в образцах после LTD-испытаний при $134^{\circ} \mathrm{C}$ и давление 0.2 МПа определили в узком диапазоне углов (от $26^{\circ}$ до $33^{\circ}$ по $2 \theta$ ), аналогично [12] по формуле:

$$
f_{m}=\frac{I_{m}(11 \overline{1})+I_{m}(111)}{I_{m}(11 \overline{1})+I_{m}(111)+I_{t}(101)+I_{c}(111)},
$$

где $I$ - это интегральная интенсивность, а индексы $m, t$ и $c$ указываю на моноклинную, тетрагональную и кубическую фазы, соответственно.

Содержание фаз и параметры их кристаллической структуры после LTD-испытаний при $180^{\circ} \mathrm{C}$ и давлении 1 МПа уточняли по результатам РФА в диапазоне углов от $23^{\circ}$ до $97^{\circ}$ по $2 \theta$.

Микроструктуру образцов изучали с помощью просвечивающей и сканирующей просвечивающей электронной микроскопии (ПЭМ и СПЭМ, соответственно) на установке JSM-2100F (JEOL, Япония) после пробоподготовки, которую осуществляли методом ионного травления на установке Ion Slicer (JEOL, Япония). Средний условный размер зерна $D$ определяли методом подсчета пересечений зерен.

Без изменения типа решётки при преобразовании базисного вектора из пространственной группы (137) в координаты пространственной группы (225), численные значения двух параметров решетки изменятся в корень из двух раз и составят набор величин, которые позволяют корректно представить степень тетрагональности простым отношением $c / a$. Аналогичный прием используется авторами в ряде работ [18-20].

Таким образом, степень тетрагональности $c / a$, наряду со сведениями о содержании фаз, может использоваться для сопоставления и поиска закономерных связей между режимами спекания, размерами структурных элементов керамики и параметрами её кристаллической структуры с результатами LTD.

\section{3. Результаты и обсуждение}

Представленные в Табл. 1 результаты анализа относительной плотности $\rho$ и среднего размера зерен $D$ спечённой керамики показывают, что добавление нановолокон $\mathrm{Al}_{2} \mathrm{O}_{3}$ способствует интенсификации усадки и более интенсивному росту зерна при одинаковых температурах спекания $T_{\text {сп }}\left(T_{\sin }\right)$, при этом, позволяя уже при температуре $1400^{\circ} \mathrm{C}$ получить керамику с зерном

Табл. 1. Плотность и средний размер зерна исследуемой керамики, спечённой при различной температуре.

Table 1. Density and average grain size of the studied ceramics sintered at different temperatures.

\begin{tabular}{|c|c|c|c|c|}
\hline \multirow{2}{*}{$\begin{array}{c}T_{\text {crn }},{ }^{\circ} \mathrm{C} \\
T_{\text {sin }},{ }^{\circ} \mathrm{C}\end{array}$} & $\rho$ & $\begin{array}{c}|c| \\
\mathrm{D}, \mathrm{HM}\end{array}$ & \multicolumn{2}{c|}{$\mathrm{Y}-\mathrm{TZP}+\mathrm{Al}_{2} \mathrm{O}_{3}$} \\
\cline { 2 - 5 } & $\rho, \mathrm{nm}$ & $\rho$ & $\begin{array}{c}D, \mathrm{HM} \\
D, \mathrm{~nm}\end{array}$ \\
\hline 1350 & $0.75 \pm 0.01$ & - & $0.97 \pm 0.01$ & - \\
\hline 1400 & $0.94 \pm 0.01$ & $182 \pm 26$ & $0.98 \pm 0.01$ & $196 \pm 21$ \\
\hline 1450 & $0.96 \pm 0.01$ & $223 \pm 23$ & $0.99 \pm 0.01$ & $244 \pm 21$ \\
\hline 1500 & $0.97 \pm 0.01$ & $256 \pm 26$ & $0.99 \pm 0.01$ & $305 \pm 19$ \\
\hline
\end{tabular}

196 нм и с относительной плотностью 98\%. На образцах без нановолокон значение плотности приближается к 98\% лишь при $1500^{\circ} \mathrm{C}$, а средний размер зерна при этом составляет 256 нм. Плотность 99\% на образцах с нановолокнами достигнута при температуре $1450^{\circ} \mathrm{C}$, а средний размер зерна при этом составляет 244 нм.

Проведённые нами испытания на стойкость к LTD при $134^{\circ} \mathrm{C}$ длительностью 40 ч показывают, что в керамике, изготовленной без добавки нановолокон при температуре спекания $1500^{\circ} \mathrm{C}$, образуется более $60 \% \mathrm{~m}$-фазы (Рис. 1). Аналогичные результаты были получены в независимых исследованиях [1], что позволяет определить температуру спекания $1450^{\circ} \mathrm{C}$ условной границей, после спекания при которой керамика Y-TZР катастрофически теряет стойкость к LTD. Напротив, образцы с добавкой нановолокон $\mathrm{Al}_{2} \mathrm{O}_{3}$, изготовленные спеканием при $1500^{\circ} \mathrm{C}$, практически не проявляют признаков старения даже при среднем размере зерна более 300 нм: содержание моноклинной фазы после LTD-испытаний не превышает 3.5 об.\% в диапазоне температур спекания от $1400^{\circ} \mathrm{C}$ до $1500^{\circ} \mathrm{C}$. Таким образом, введение в Y-TZР нановолокон $\mathrm{Al}_{2} \mathrm{O}_{3}$ позволяет повысить стойкость спечённой при высоких температурах керамики к LTD. Предположительно, повышение стойкости к LTD может быть обусловлено сегрегацией ионов $\mathrm{Al}$ на границах зерен Y-TZP $[2,12]$, однако наши исследования, проведённые методом энергодисперсионной спектроскопии, не подтверждают этого предположения.

Поскольку в стандартных условиях испытаний керамики с нановолокнами достоверная оценка столь малых (до 4\%) изменений содержания $m$-фазы затруднительна, дальнейшие испытания на стойкость к LTD проводили при $180^{\circ} \mathrm{C}$ и давлении 1 МПа. В Табл. 2 представлены результаты РФА: исходного (до LTDиспытаний) фазового состава, содержание $m$-фазы после 20 часов старения, параметры $a$ и $c$ решёток соответствующих фаз, а также величины их отношения c/a (степени тетрагональности).

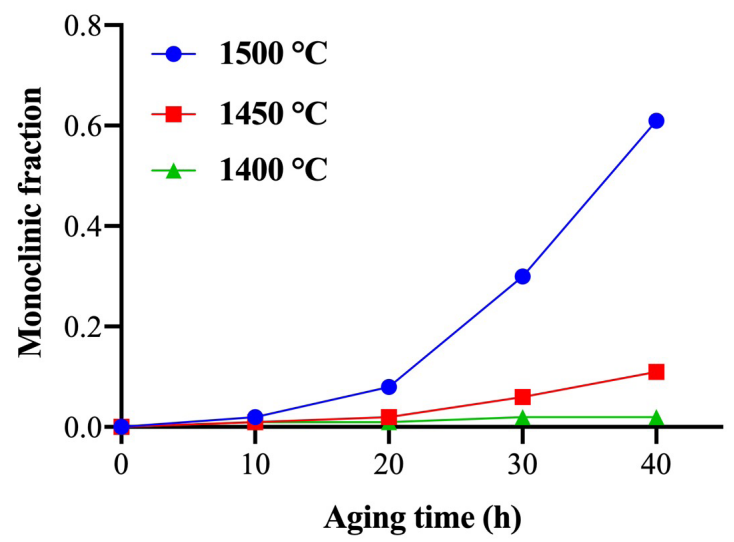

Pис. 1. (Color online) Зависимости содержания моноклинной фазы в образцах Y-TZP, спеченных при $1400^{\circ} \mathrm{C}, 1450^{\circ} \mathrm{C}$ и $1500^{\circ} \mathrm{C}$ от времени старения в водяном паре при $134^{\circ} \mathrm{C}$ и давлении $0.2 \mathrm{MПа.}$

Fig. 1. (Color online) Dependences of the content of the monoclinic phase in the Y-TZP samples, sintered at $1400^{\circ} \mathrm{C}, 1450^{\circ} \mathrm{C}$ and $1500^{\circ} \mathrm{C}$, on the aging time in water vapor at $134^{\circ} \mathrm{C}$ and pressure of $0.2 \mathrm{MPa}$. 
Изучение исходного фазового состава керамических образцов, спечённых в диапазоне температур от $1350^{\circ} \mathrm{C}$ до $1600^{\circ} \mathrm{C}$, показало, что наиболее достоверные (с минимальным параметром ошибки $R_{\text {wp }}$ ) результаты обработки дифрактограмм достигаются в предположении о наличии в этих материалах двух типов тетрагональных фаз ( $t_{1}$ и $\left.t_{2}\right)$, отдельно учитываемых по условному критерию: фаза $t_{1}-$ с низкой степенью тетрагональности $(c / a<1.0141)$ и фаза $t_{2}-$ с высокой степенью тетрагональности $(c / a>1.0141)$.

Результаты исследования кристаллической структуры показали, что при температурах спекания $1350^{\circ} \mathrm{C}$ и $1400^{\circ} \mathrm{C}$ керамики Y-TZP + 1 об.\% $\mathrm{Al}_{2} \mathrm{O}_{3}$ и Y-TZP, соответственно, степени тетрагональности их фаз $t_{1}$ и $t_{2}$ практически одинаковы. Увеличение температуры спекания приводит к увеличению отношения $c / a$ для $t_{1}$-фазы и снижению отношения $c / a$ для $t_{2}$-фазы. По мере повышения температуры спекания керамики Y-TZP степень тетрагональности её $t_{2}$-фазы уменьшается, а при температуре спекания $1600^{\circ} \mathrm{C}$ достигает минимального значения $(c / a=1)$, что позволяет отнести её к кубической модификации.

После LTD-испытаний образцов Y-TZP, спечённых при $1500^{\circ} \mathrm{C}$, установлено полное отсутствие $t_{1}$-фазы, что косвенно указывает на минимальную стойкость к деградации именно этой фазы и на её повышенную склонность к переходу в моноклинную модификацию под действием неблагоприятных факторов (Рис. 3).

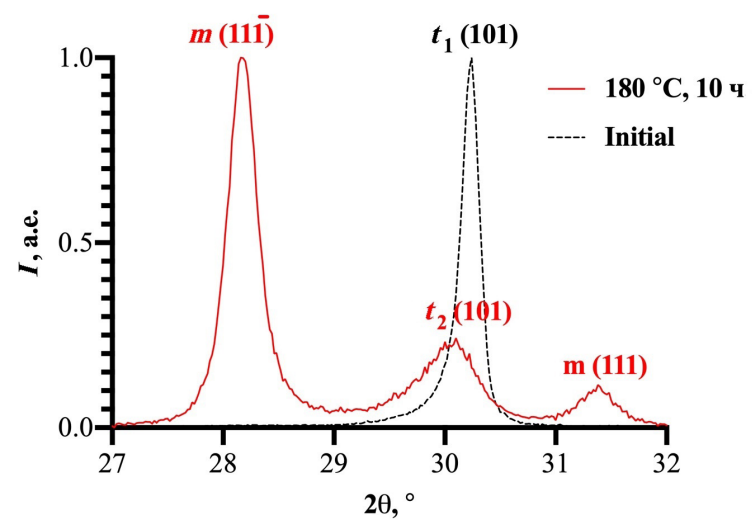

Pис. 3. (Color online) Дифрактограммы образца Y-TZP, спеченного при $1500^{\circ} \mathrm{C}$, до и после старения в водяном паре при $180^{\circ} \mathrm{C}$ длительностью 10 ч.

Fig. 3. (Color online) X-ray diffraction patterns of Y-TZP sample sintered at $1500^{\circ} \mathrm{C}$ before and after aging in water vapor at $180^{\circ} \mathrm{C}$ for $10 \mathrm{~h}$.

табл. 2. Результаты уточнения структуры методом Ритвельда исследуемых образцов.

Table 2. The results of structure refinement by Rietveld method of investigated samples.

\begin{tabular}{|c|c|c|c|c|c|c|c|c|c|c|c|}
\hline \multirow{2}{*}{$\begin{array}{c}\text { Материал } \\
\text { Material }\end{array}$} & \multirow{2}{*}{$\begin{array}{l}T_{\mathrm{cr}},{ }^{\circ} \mathrm{C} \\
T_{\text {sin }},{ }^{\circ} \mathrm{C}\end{array}$} & \multicolumn{4}{|c|}{$\begin{array}{l}t_{1} \text {-фаза } \\
t_{1} \text {-phase }\end{array}$} & \multicolumn{4}{|c|}{$\begin{array}{c}t_{2} \text {-фаза } \\
t_{2} \text {-phase }\end{array}$} & \multirow{2}{*}{$R_{\mathrm{wp}}$} & \multirow{2}{*}{ 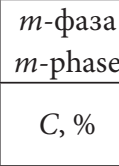 } \\
\hline & & $C, \%$ & $\begin{array}{l}a, \mathrm{HM} \\
a, \mathrm{~nm}\end{array}$ & $\begin{array}{l}c, \mathrm{HM} \\
c, \mathrm{~nm}\end{array}$ & $c / a$ & $C, \%$ & $\begin{array}{l}a, \mathrm{HM} \\
a, \mathrm{~nm}\end{array}$ & $\begin{array}{l}c, \mathrm{HM} \\
c, \mathrm{~nm}\end{array}$ & $c / a$ & & \\
\hline \multirow{5}{*}{ Y-TZP } & 1400 & 44 & 0.50972 & 0.51720 & 1.0147 & 56 & 0.51028 & 0.51749 & 1.0141 & 12.49 & 6 \\
\hline & 1450 & 60 & 0.50964 & 0.51720 & 1.0148 & 40 & 0.51100 & 0.51766 & 1.013 & 16.53 & 25 \\
\hline & 1500 & 62 & 0.50981 & 0.51758 & 1.0152 & 38 & 0.51192 & 0.51763 & 1.0112 & 16.31 & 75 \\
\hline & 1550 & 76 & 0.50977 & 0.51775 & 1.0157 & 24 & 0.51237 & 0.51610 & 1.0073 & 15.11 & - \\
\hline & 1600 & 67 & 0.50967 & 0.51769 & 1.0157 & 33 & 0.51352 & 0.51352 & 1 & 15.36 & - \\
\hline \multirow{4}{*}{$\begin{array}{c}\mathrm{Y}-\mathrm{TZP}+ \\
1 \text { об.\% } \mathrm{Al}_{2} \mathrm{O}_{3} \\
\mathrm{Y}-\mathrm{TZP}+ \\
1 \text { vol. } \% \mathrm{Al}_{2} \mathrm{O}_{3}\end{array}$} & 1350 & 24 & 0.50994 & 0.51729 & 1.0144 & 76 & 0.51033 & 0.51756 & 1.0142 & 11.52 & 2 \\
\hline & 1400 & 58 & 0.50989 & 0.51733 & 1.0146 & 42 & 0.51100 & 0.51756 & 1.0128 & 19.28 & 17 \\
\hline & 1450 & 78 & 0.50940 & 0.51700 & 1.0149 & 22 & 0.51115 & 0.51712 & 1.0117 & 15.17 & 57 \\
\hline & 1500 & 58 & 0.50974 & 0.51770 & 1.0156 & 42 & 0.51607 & 0.51770 & 1.0031 & 16.77 & 74 \\
\hline
\end{tabular}

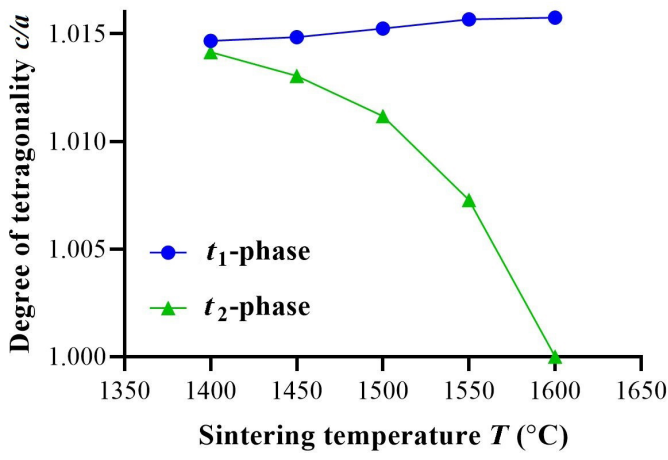

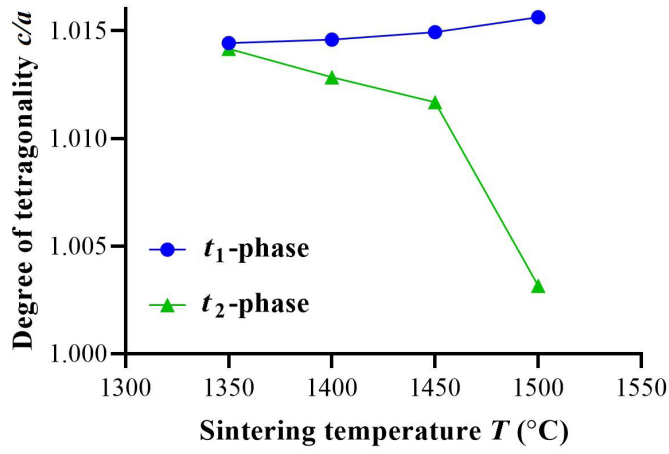

b

Pис. 2. (Color online) Зависимость степени тетрагольности $c / a t_{1}$ и $t_{2}$ фаз от температуры спекания $T_{\text {сп }}$ : Y-TZP (а), $\mathrm{Y}-\mathrm{TZP}+1$ об. $\% \mathrm{Al}_{2} \mathrm{O}_{3}(\mathrm{~b})$.

Fig. 2. (Color online) Dependence of the degree of tetragonality $c / a$ of $t_{1}$ and $t_{2}$ phases on the sintering temperature $T_{\sin }$ : Y-TZP (a), $\mathrm{Y}-\mathrm{TZP}+1$ vol.\% $\mathrm{Al}_{2} \mathrm{O}_{3}(\mathrm{~b})$. 


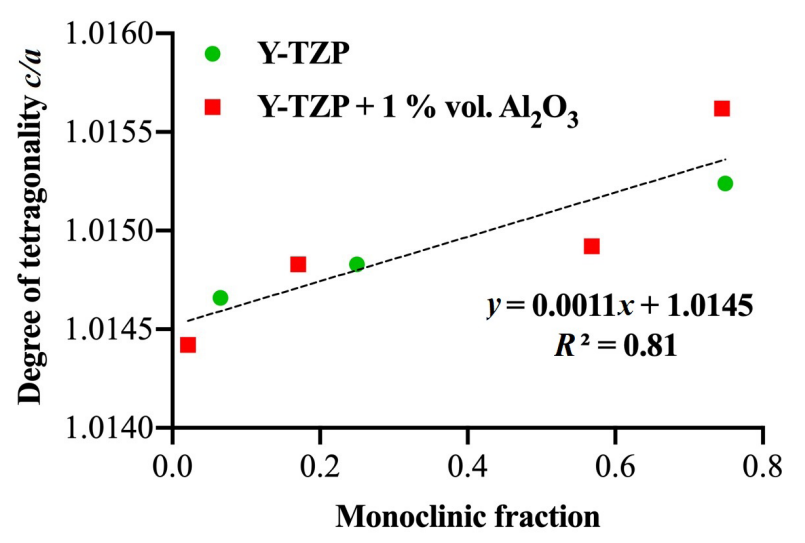

Pис. 4. (Color online) Поле корреляции $c / a t_{1}$-фазы исходных (до испытаний) керамических материалов и содержания $m$-фазы в них после старения в водяном паре при $180^{\circ} \mathrm{C}$ и давлении 0.2 МПа в течении 20 ч.

Fig. 4. (Color online) The correlation field of the $c / a t_{1}$-phases of the initial (before testing) ceramics materials and the $m$-phase content in them after aging in water vapor at $180^{\circ} \mathrm{C}$ and a pressure of $0.2 \mathrm{MPa}$ for $20 \mathrm{~h}$.

На Рис. 4 представлено поле корреляции $c / a$ $t_{1}$-фазы исходных (до испытаний) керамических материалов от содержания $m$-фазы после испытаний при $180^{\circ} \mathrm{C}$ и давлении 1 МПа. Содержание $m$-фазы линейно (с достоверностью $81 \%$ ) увеличивается по меpe увеличения степени татрагональности $t_{1}$-фазы. Обнаруженная закономерность позволяет заключить, что в принятых условиях LTD фазовый состав керамики остаётся стабильным при степени тетрагональности не более 1.0145, а превышение этого значения на 0.0011 (до 1.0156) может привести к тотальному тетрагонально-моноклинному переходу (к полной деградации).

\section{4. Выводы}

Добавление в коммерческий порошок TZ-3YS 1 об.\% метастабильных нановолокон $\mathrm{Al}_{2} \mathrm{O}_{3}$ позволяет из компактов, изготовленных одноосным односторонним прессованием в закрытой жесткой пресс-форме, уже при температуре $1400^{\circ} \mathrm{C}$ получить керамику с зерном 196 нм и с относительной плотностью $98 \%$. На образцах без нановолокон значение плотности приближается к $98 \%$ лишь при $1500^{\circ} \mathrm{C}$ и сопровождается увеличением среднего размера зерна на $25 \%$.

Образцы с добавкой нановолокон $\mathrm{Al}_{2} \mathrm{O}_{3}$, изготовленные спеканием при $1500^{\circ} \mathrm{C}$, практически не проявляют признаков старения при $134^{\circ} \mathrm{C}$ даже при среднем размере зерна более 300 нм: содержание моноклинной фазы после LTD-испытаний не превышает 3.5\%. При этом содержание моноклинной фазы в образце Y-TZP (без добавления нановолокон $\mathrm{Al}_{2} \mathrm{O}_{3}$ ), изготовленного в аналогичных условиях, превышает $60 \%$, а средний размер зерна составляет 256 нм.

Установлена закономерная связь степени тетрагональности исходного материала с образованием в нём после испытаний на LTD моноклинной фазы, позволяющая заключить, что в принятых условиях LTD фазовый состав исследуемой керамики остаётся стабильным при степени тетрагональности не более 1.0145, а превышение этого значения на 0.0011 (до 1.0156) может привести к тотальному тетрагонально-моноклинному переходу (к полной деградации). Обнаруженная закономерность расширяет возможности для оптимизации режимов изготовления и достижения требуемого баланса различных эксплуатационных свойств керамики Y-TZP, используемой в качестве имплантатов или ответственных изделий, работающих в агрессивных условиях и влажной среде.

Благодарности/Acknowledgements. Исследование выполнено на базе "Нано-Центра» Томского политехнического университета при финансовой поддержке РФФИ в рамках научного проекта № 18-33-00763 и Госзадания “Наука" №5.0017.ГЗБ.2020. Изготовление образиов для исследований и их аттестация методами рентгенобазового анализа выполнены при поддержке Госзадания "Наука". Испытание керамики на стойкость $\kappa$ LTD выполнено при поддержке РФФИ (проект №18-33-00763). / The study was carried out at the Innovation Centre for Nanomaterials and Nanotechnologies of Tomsk Polytechnic University. The reported study was funded by RFBR according to the research project No. 18-33-00763 and State tasks "Science" No. 5.0017.GZB.2020. The production of samples for research and their attestation by X-ray phase analysis was performed with the support of the State tasks "Science". The ceramic test for resistance to LTD was carried out with the support of the RFBR (Project No. 18-33-00763).

\section{Литература/References}

1. S. Ramesh, K. Y. Sara Lee, C. Y. Tan. Ceram. Int. 44 (17), 20620 (2018). $\underline{\text { Crossref }}$

2. F. Zhang, K. Vanmeensel, M. Batuk, J. Hadermann, M. Inokoshi, B. Van Meerbeek, I. Naert, J. Vleugels. J. Eur. Ceram. Soc. 35 (2), 741 (2015). Crossref

3. S. Sequeira, M.H. Fernandes, N. Neves, M. M. Almeida. Ceram. Int. 43 (1), 693 (2017). $\underline{\text { Crossref }}$

4. G. Sreedhar, M. Alam, V.S. Raja. Surf. Coat. Technol. 204 (3), 291 (2009). Crossref

5. M. Saremi, Z. Valefi, N. Abaeian. Surf. Coatings Technol. 221, 133 (2013). Crossref

6. O. Vasylkiv, Y. Sakka, V. V. Skorokhod. J. Am. Ceram. Soc. 86 (2), 299 (2003). Crossref

7. B.D. Flinn, A.J. Raigrodski, A. Singh, L.A. Mancl. J. Prosthet. Dent. 112 (6), 1377 (2014). Crossref

8. B. Basu, J.H. Lee, D. Y. Kim. J. Am. Ceram. Soc. 87 (9), 1771 (2004). Crossref

9. S. A. Salehi, K. Vanmeensel, A. K. Swarnakar, O. Van der Biest, J. Vleugels. J. Alloys Compd. 495 (2), 556 (2010). $\underline{\text { Crossref }}$

10. A. Smirnov, H.D. Kurland, J. Grabow, F. A. Müller, J. F. Bartolomé. J. Eur. Ceram. Soc. 35 (9), 2685 (2015). Crossref

11. Z. K. Wu, N. Li, C. Jian, W. Q. Zhao, J.Z. Yan. Ceram. Int. 39 (6), 7199 (2013). rossref 
12. K. Matsui, H. Yoshida, Y. Ikuhara. Sci. Rep. 4 (1), 4758 (2015). Crossref

13. R. Chintapalli, A. Mestra, F. G. Marro, H. Yan, M. Reece, M. Anglada. Materials (Basel). 3 (2), 800 (2010). Crossref

14. K. Matsui, N. Ohmichi, M. Ohgai, H. Yoshida, Y. Ikuhara. J. Mater. Res. 21 (9), 2278 (2006). Crossref

15. O.S. Tolkachev, E.S. Dvilis, T.R. Alishin, A.A. Leonov, V.D. Paygin, D.A. Mikheev. Composite materials constructions. 3, 30 (2019). (in Russian) [O. С. Толкачёв, Э. С. Двилис, Т. Р. Алишин, А. А. Леонов, В. Д. Пайгин, и Д.А. Михеев. Конструкции из композиционных материалов. 3, 30 (2019).]

16. ISO standard 13356:2008. Implants for surgery: ceramic materials based on yttria- stabilized tetragonal zirconia
(Y-TZP). Geneva, Switzerland: ISO. Available at: http://www.iso.ch/iso/en/prods-services/ISOstore/store. html (Last accessed September 22, 2014).

17. Japanese Standards Association. JIS A5207-2010 Sanitary wares. Tokyo, Japan: Japanese Standards Association. Available at: http://www.jsa.or.jp. (Last accessed September 22, 2014.)

18. K. Muraleedharan, J. Subrahmanyam, S. B. Bhaduri. J. Am. Ceram. Soc. 71 (5), 226 (1988). Crossref

19. H. G. Scott. J. Mater. Sci. 10 (9), 1527 (1975). Crossref

20. G. Ya. Akimov, G. A. Martini, V. Yu. Kameneva. Solid state physics. 46 (2), 250 (2004). (in Russian) [Г.Я. Акимов, Г.А. Маринин, и В.Ю. Каменева. Физика твердого тела. 46 (2), 250 (2004).] 